\title{
OCULAR PROPRIOCEPTION AND EFFERENCE COPY IN REGISTERING VISUAL DIRECTION
}

\author{
Bruce Bridgeman and LaWrence Stark \\ Program in Experimental Psychology, Clark Kerr Hall, University of California-Santa Cruz, \\ Santa Cruz, CA 95064 and Neurology Unit, 481 Minor Hall, University of California-Berkeley, \\ Berkeley, CA 94720, U.S.A.
}

(Received 18 June 1990; in revised form 1 March 1991)

\begin{abstract}
We measured the roles of eye muscle proprioception ("inflow") and efference copy ("outflow") in registering eye position. During monocular fixation, pressing on the side of an occluded eye results in a passive rotation, changing the proprioception without affecting oculomotor efference. As we have shown previously, a constant press on the side of the viewing eye induces active resistance to rotation, changing efference because oculomotor innervation compensates for the eyepress; the viewing eye's fixation remains constant. Using these two types of eyepress, both perceived target deviations and pointing biases in an unstructured visual field were measured in 8 subjects under efference copy, proprioception and control (no eyepress) conditions. Eye deviation was measured photoelectrically. Physiological gains of efference copy and proprioception were about $5 / 8$ and $1 / 4$ respectively. There was no statistically significant difference between perceptual judgement and open-loop pointing. The sum of gains of efference copy and proprioception, about $7 / 8$, indicates incomplete registration of eye eccentricity in an unstructured field, and quantitatively accounts for several previously unexplained results in the literature.
\end{abstract}

Inflow Outflow Efference copy Corollary discharge Eyepress Proprioception

To locate an object in visual space, both the position of the image on the retina and the position of the eye must be known. The position of the eye relative to the head might be determined either from mechanoreceptors in the muscles controlling the position of the eye (an oculomotor proprioception or "inflow" from the periphery to the brain), or from internal monitoring of the innervations sent to those muscles (an efference copy or "outflow" from the brain to the periphery). The relative contributions of proprioception and efference copy remain unknown.

\section{Efference copy}

Following the lead of Descartes $(1665,1972)$, efference copy was the dominant candidate for extraretinal signals in the 19th century (Helmholtz, 1866; Hering, 1868, 1977). The preponderance of psychophysical and behavioral evidence has implicated efference copy as the signal used in perception and visually guided behavior. von Holst and Mittelstaedt (1950) rotated the head of a fly and found forced circling that could be accounted for by an "efference copy"; Sperry (1950) made similar observations of circling in a fish with inverted eyes, with a similar interpretation in terms of "corollary discharge".

Following up on Helmholtz's (1866) observations, paralysis studies have implicated efference copy in determining perceived position. During paralysis, subjects perceive motions of the world at the time of attempted eye movements despite lack of proprioceptive input (Siebeck, 1954; Kornmüller, 1931; Brindley, Goodwin, Kulikowski \& Leighton, 1976; Stevens, Emerson, Gerstein, Kallos, Neufeld, Nichols \& Rosenquist, 1976). The paralysis studies of Matin, Picoult, Stevens, Edwards, Young and MacArthur $(1980,1982)$ also show that visual context plays an important role in perceived position even when extraretinal signals are mismatched with gaze position; the mismatch results in perceptual mislocalizations only in an unstructured visual field. Using the eyepress method, Bridgeman and Graziano (1989) have shown recently that with sensitive measurements there are indeed effects of the mismatch even in a structured field, but they are smaller than in an unstructured field.

Other experiments using the method of pressing on the side or lower lid of the eye have clarified relative contributions of propriocep- 
tion and efference copy signals under conditions where the two theories make different predictions. When a subject presses slowly on the side of one eye, while the other is occluded, oculomotor stabilization systems maintain fixation on the current fixation point. As a result, the innervation to the eye changes, but the posture of the eye does not (Bridgeman, 1979). Rather, the eye is displaced sideways in the orbit. This analysis has been supported by several kinds of experiments, using both i.r. oculography (Stark \& Bridgeman, 1983) and the search coil technique (Ilg, Bridgeman \& Hoffman, 1989).

Now if efference copy determines visual localization with eyepress, the amount of perceptual deviation should match the amount of the deviation in efference, which can be measured quantitatively by monitoring the position of the occluded fellow eye. Only one eye moves, but both receive an equal innervation. If proprioception determines localization in the monocular eyepress, however, the situation is different. The proprioceptive signals from the rotated, occluded eye and the pressed, fixating eye should sum according to Hering's law: preceptual offset should be $1 / 2$ of eye deviation, and the slope of a line relating eye deviation to perceptual offset should be 0.5 . But measured perceptual deviation in nearly as great as the eye deviation, implicating a dominance of efference copy (Stark \& Bridgeman, 1983; Bridgeman \& Delgado, 1984; Bridgeman \& Fishman, 1985). The slope of the line relating perceptual deviation to eye deviation is $<1$, however, indicating that other influences may participate in internal monitoring of eye position.

Another observation that supports efference copy is that the retina is more accurate than any plausible proprioceptive signal, for proprioception would be available only after a transduction and conduction delay. Therefore it is assumed that proprioception serves only to register static muscle length or tension; no feedback can be provided during a saccade.

\section{Oculomotor proprioception}

Growing knowledge of muscle receptors led Sherrington $(1898,1918)$ to propose that proprioception from the extraocular muscles was important to spatial localization. Several kinds of receptors are found in various mammalian eyes, including stretch receptors (Bach-y-Rita \& Ito, 1966) and palisade endings (Steinbach \& Smith, 1981) near the distal insertions of the extraocular muscles. Physiological recording shows that proprioceptive information reaches superior colliculus (Donaldson \& Long, 1980) and visual cortex (Buisseret \& Maffei, 1977) among other regions.

More recently, Ashton, Boddy and Donaldson (1984) have found that most single units in the cat striate cortex are sensitive to specific directions of passive eye movement, and Ashton, Boddy, Donaldson and Milleret (1985) found direct ocular proprioceptive input to the vestibular nuclear complex and the reticular formation of the medulla in the cat. Steinbach (1987) and Milleret (1987) review the anatomy and physiology, concluding that proprioception provides an important, if small, contribution to the extraretinal eye position signal.

Clearly, such an elaborate physiological system should have a functional role. What then is the role of the proprioceptors? Current evidence implicates them in low-level gaze stabilization and assigns them a role in calibrating the system during development. In cats, cutting proprioceptive fibers in the ophthalmic branch of the $V$ th nerve results in gaze instability when the animals are in darkness and cannot stabilize fixation with retinal feedback (Fiorentini \& Maffei, 1977). A similar intervention in rabbits results in large tonic eye deviations during vestibular nystagmus, even while the unoperated contralateral eye shows the same fast phase beats of nystagmus and good positional control (Kashii, Matsui, Honda, Sasa \& Takaori, 1989). It has also been proposed that proprioception serves as a parametric feedback signal in maintaining gaze position (Ludvigh, 1952), and Skavenski (1972) reported that highly practiced subjects could use inflow under limited conditions.

Other evidence supports a developmental role for oculomotor proprioception. Hein and Diamond (1983) surgically interrupted proprioception in cats and found deficits in visually guided behavior, but only in developing animals. The same interruptions in adult cats had no effect on visual-motor coordination. Trotter, Fregnac and Buisseret (1987) reached a similar conclusion regarding the development of binocularity in cats. The binocularity effect in turn meant that muscle proprioception was necessary for the development of normal depth perception (Graves, Trotter \& Fregnac, 1987).

A recent study by Gauthier, Nommay and Vercher $(1990 \mathrm{a}, \mathrm{b})$ has shown an important effect of proprioception on visually guided behavior. Gauthier et al. separated gaze pos- 
ition from innervation with a contact lens method similar to that of Robinson (1964) and of Skavenski, Haddad and Steinman (1972). A suction contact lens was equipped with a small stalk, which could be clamped while the subject was gazing eccentrically. When the subject attempted to turn the gaze to another position, the clamped eye remained in place while the other eye moved to the intended position. Cook and Stark (1968) modeled the eye muscle forces in this situation and showed that no alteration of the control force program occurred; this eye movement control was open-loop to both vision and proprioception during a saccade. Measuring open-loop pointing, Gauthier et al. (1990a, b) obtained a shallow slope of proprioception effects and a threshold below which changes in inflow had no effect on behavior.

The experiments reported here confirm our earlier efference copy experiment (Stark \& Bridgeman, 1983) with eye press (on the viewing eye). In the same paradigm we confirm Gauthier's effect with eye press on the covered eye, allowing a quantitative comparison between the proprioception and efference copy effects.

\section{METHODS}

The method depends upon an extension of the monocular eyepress method of Bridgeman and Stark (1981) and of Stark and Bridgeman (1983) to separate gaze position from innervation state. As described in those papers, the method begins with a reanalysis of the traditional interpretation of apparent motion observed during a press on the outer canthus of the eye; instead of attributing the apparent motion to passive movement of the eye, the new analysis recognizes an active resistance of the fixating eye to the rotatory force of the finger. As a subject presses harder and harder on the fixating eye, the oculomotor system adds more and more compensatory innervation to maintain the position of a target on the retina. It is this increase in innervation, rather than a rotation of the eye, that causes the perception of motion during eyepress; experiments have shown that the viewing eye does not rotate for a slow eyepress (Ilg et al., 1989). Thus conditions similar to those during paralysis occur: oculomotor innervation changes while position of the image on the retina remains nearly stable. A sustained press on the viewing eye thus changes efference copy without changing fixation. On the other hand, pressing on the occluded eye changes ocular posture (and with it, proprioceptive inflow) without affecting efference copy. The latter technique as a way of influencing proprioception has been suggested independently by Mittelstaedt (1990, p. 282).

\section{Apparatus}

Subjects sat before a uniform hemicylindrical screen $180 \mathrm{deg}$ wide, at a distance of $60 \mathrm{~cm}$. The head was fixed in place by a bite bar of dental wax. On the screen a small target spot, $0.5 \mathrm{deg}$ in dia., was projected from a tungsten halogen lamp. A galvanic mirror in the optical path allowed the spot to be moved horizontally under computer control. A computer-controlled mechanical shutter was opened to begin each trial.

The spot was projected at one of 5 possible locations, 2 deg apart, with the third of the 5 positions at the centerline of the screen. Vertical position of the target was $29 \mathrm{deg}$ above eye level, a position of gaze where most subjects can establish a consistent horizontal apparent motion from eyepress, with minimal vertical motion. For calibration, subjects fixated a target centered on the midline and $29 \mathrm{deg}$ above eye level, flanked by two additional targets $5 \mathrm{deg}$ left and right of the center target respectively. The position of the photocells of the monitor was raised to obtain an optimal signal at this elevated gaze position, and adjusted so that fixations of the left and right targets yielded equal signals.

Perceptual responses were recorded on a keyboard, and pointing was performed with a pointer having its center of rotation near the center of curvature of the screen. The pointer was mounted on a potentiometer that sent a variable voltage to the $a / d$ converter of the computer using a simple analog circuit. We used two measures because of evidence that they access two different internal representations of visual space (Bridgeman, Kirch \& Sperling, 1981; Paillard, 1987; Wong \& Mack, 1981).

\section{Design}

Subjects were tested in three conditions. First was a control condition: viewing through the right eye, the subject performed a 5-alternative judging task or a pointing task. This provided a baseline for evaluation of the effects of the other two conditions.

The second condition was designed to measure the effects of changed oculomotor 
efference copy on the tasks. The right eye was occluded, and the subject pressed the left eye. Then the shutter was opened, and the subject performed either the judging or the pointing task while holding the eye and fixating the target. The added oculomotor innervation required to compensate for the rotary effects of the eyepress also changed the efference to the eye, and thus changed the efference copy as well. But gaze position remained the same, controlled by the fixation on the target (Fig. 1, left). Thus proprioception from receptors signalling muscle length, or from extramuscular receptors, would remain the same as in the control condition for the viewing eye.

In this condition the fellow eye rotates even though it is occluded, because the changed oculomotor innervation affects both eyes equally according to Hering's law (Hering, $1867,1977)$. The proprioceptive inflow from the occluded eye should change accordingly. Thus, in this condition both proprioception and efference copy will change, but by different amounts. The change in efference copy will equal the deviation of the occluded eye, because both eyes are innervated equally, but the proprioception change will affect only the occluded eye. The binocular proprioception signal will be the average of the proprioception signals from the two eyes; the unchanged proprioception from the viewing eye will combine with the proprioception from the deviated occuluded eye to yield a

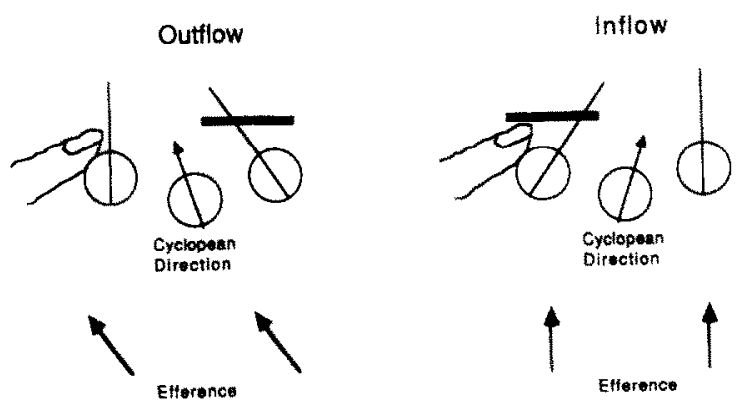

Fig. 1. Analysis of efference copy (Outflow, left) and proprioception (Infiow, right) conditions, seen from above. In each case the left eye is pressed steadily with the subject's left forefinger. Left and right circles in each drawing represent the eyes; the third circle between and slightly behind the eyes represents the cyclopean eye, situated on the Veith-Müller horopter. The cyclopean eye's proprioceptively given position is the average of the gaze positions of the two eyes, and its centerline represents the best estimate of the composite proprioception signal. Clockwise rotational force from the finger causes the innervation state of the pressed eye to deviate from its gaze posture when a visual target is present (left), but results in a passive rotation when the pressed eye is occluded (right) composite inflow of half the monocular deviation.

This analysis assumes that a single direction is specified from the composite of both eyes. In strabismics the signal may originate only from the dominant eye, and alternating strabismics may possess separate extraretinal signals from the two eyes that can be switched according to the currently fixating, and non-suppressed, eye. Thus our conclusions will be limited to binocularly normal subjects.

The third condition measured the contribution of proprioceptive inflow from the eye muscles to the CNS. The left eye was occluded and pressed, while the subject performed the judging or pointing task with the right eye (Fig. 1, right). In this condition the occluded eye deviates from the fixation position, but there is no visual feedback to stimulate fixation control mechanisms to change the oculomotor innervation. Thus efference copy remains the same as in the control condition. Proprioception is changed, however, because the passive rotation of the occluded eye will stimulate proprioceptors in the extraocular muscles. Again, the composite binocular proprioception signal will equal half of the mechanical deviation of the pressed eye.

In summary, the efference copy condition (pressed eye viewing) changes both the efference copy to the eye and the proprioception from it. The inflow condition (pressed eye occluded) changes the proprioception but leaves efference copy the same as in the control condition.

\section{Procedure}

Subjects performed one of two tasks in each of the three experimental conditions, all interspersed in a modified random order. One task was a judgment of the target's position, a 5alternative forced choice decision. This task measured the effects of changes in efference copy and proprioception on visual perception. The other task was to point to the position of the target. This task measured visually guided behavior, separate from the effects on perception. Since the pointer was occluded from view at the bottom of the screen, subjects were instructed to point to the position that the spot would take if it fell straight down from the screen. Subjects found this task easy, and were not disoriented by the constant vertical distance between the target and the pointer.

Training. In order to encourage egocentric judgments of position, subjects were never 
shown more than 1 target position at a time. They were first shown the 5 possible positions of the target in sequence, repeated 3 times, on an otherwise blank screen. Then subjects underwent a series of training trials. Each trial began with a randomly selected target exposed for $1 \mathrm{sec}$. The subjects estimated the target position with one of the 5 response keys ("judging trials"), and continued until they were correct in 5 consecutive trials. Next, they were trained on pointing, with the same stimuli in a new random sequence ("pointing trials"), until they spontaneously returned the pointer to its rightmost position (as initially instructed) for 5 consecutive trials.

The tasks were cued with an auditory signal, a brief "beep" for the judging task and a "squawk" for the pointing task. During experimental runs the subjects performed one or the other of these tasks in each trial. Two tasks $\times 3$ conditions $\times 5$ target positions yielded 30 trial types. Each was repeated 5 times in a single session, for a total of 150 trials per subject. Each subject received at least one rest period during the session. The random order of trials was constrained so that judging and pointing trials of a given trial type alternated in the sequence. Thus no two consecutive trials were identical in all parameters. Each subject was run in a different computer-generated pseudorandom order of trials. Since response usually occurred within $2 \mathrm{sec}$ of the initiation of eyepress, and control trials without eyepress were interspersed, the possibility of adaptation was minimized.

Movements of the occluded eye, while the viewing eye was pressed, were recorded with an i.r. technique (Stark, Vossius \& Young, 1962). Paired i.r. photocells, mounted on the bite bar frame, were aimed at the left and right iris-sclera borders to record horizontal eye deviations. The subject viewed a target on the midline $29 \mathrm{deg}$ above eye height, to determine an average eyepress-induced ocular deviation for each subject. Eye recordings were calibrated with fixation targets $5 \mathrm{deg}$ left and $5 \mathrm{deg}$ right of the central target.

\section{Analysis}

Within-subjects analysis. Two 2-way analyses of variance were performed for each subject and each task. One tested the control vs the efference copy condition, and the other tested the control vs the proprioception condition. Factors were target position and experimental condition.
Between-subjects analysis. For each subject a mean magnitude of effect was found by subtracting control data from experimental data in each of the two experimental conditions for each of the two tasks. Using these data, between-subjects analyses were conducted with $t$-tests.

\section{Subjects}

A total of eight subjects were run; six were undergraduates and one a graduate student, all volunteers. Each received practice with the eyepress technique before beginning the experiments, so that a consistent effect could be elicited even without visual feedback. One additional subject was run for $\mathbf{8 0}$ trials in exactly the design described above. He used a different pointer, a stylus that responded with a voltage when it touched a coil of nichrome wire stretched along the base of the screen. For this subject the head was restrained from behind with a 2-point adjustable pad. In this final subject only the effects of the 3 conditions were tested statistically; because of reduced power, effects of target position and the position $x$ condition interaction were not tested statistically. This subject was recorded both in this experiment and in another study with different methods but similar goals (Gauthier et al., $1990 \mathrm{~b}$ ), allowing a direct comparison between the studies.

\section{RESULTS}

Changes in efference copy affected both pointing and judging, in ways expected from previous work (Stark \& Bridgeman, 1983; Matin et al., 1982). The effects of proprioception, in contrast, were small for both perception and visually guided behavior.

\section{Efference copy}

We first repeated our finger-press efference copy paradigm on eight subjects. Results were similar to those published previously (Stark \& Bridgeman, 1983; Bridgeman, 1986); in both the previous experiments and the current series, a press on the monocularly viewing eye yielded large and consistent deviations in both pointing and perceived direction.

Perception. When judging the target's position on the 1-5 scale, subjects showed large effects of changes in efference copy, elicited by pressing on the viewing eye. Deviations from control judgements average $3.8^{\circ}$. Analyses of variance showed that the effect of eyepress was significant in all eight subjects $(P=0.0032$ or 
less in each). As expected, perceived position was also significantly affected by target position $(P<0.001$ in each subject $)$.

Of the 28 analyses of variance performed for all conditions, one showed a significant row $x$ column interaction at a criterion of $P<0.01$. Slope of the least-squares linear regression of perceptual deviation on eye deviation was 0.74 , and correlation between the two variables was $r=0.79$ (Fig. 2, top).

Open-loop pointing. Results for open-loop pointing were similar to those for the perceptual measure; the average deviation of pointer positions from those in the control condition was $3.4^{\circ}$. This is the expected result from previous work, since a visual context was not present for any of the conditions in the present experiments.

\section{Proprioception}

By shifting the cover to the pressed eye we approximated the Gauthier et al. (1990a, b) paradigm. As noted previously (Stark \& Bridgeman, 1983, p. 373), there was negligible resistance to the eyepress in this condition. We found similar results to Gauthier's effect in the influence of eyepress on visual direction.

Perception. In separate within-subjects analyscs, proprioception had statistically significant perceptual effects in only two of the

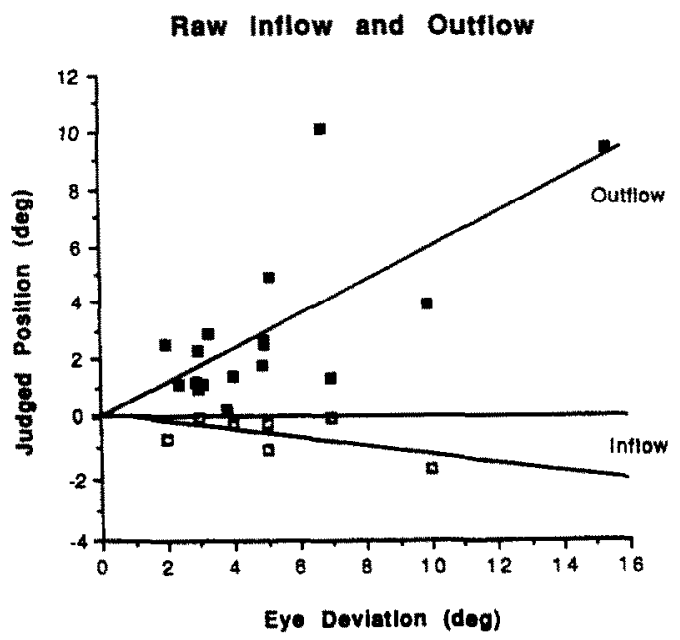

Fig. 2. Data from the efference copy (Outfow, solid symbols) and proprioception (Inflow, open symbols) judging conditions vs rotation of the occluded eye measured with i.r. oculography. Each subject is represented by one point. Eye rotation data were unavailable for one subject. Efference copy data are combined with the data of Bridgeman and Fishman (1985), collected under identical conditions. An equal contribution of both variables would result in a line through the origin with a slope of 1 . Diagonal lines are least-squares regression lines for the two data sets. eight subjects. These were the two with the largest measured amounts of proprioceptive influence. In these two subjects we could test a prediction elaborated above--that if proprioception and efference copy were equally effective, the deviations in the proprioception condition should equal half of those in the efference copy condition in our design. In both cases, the proprioception fell short of half of the efference copy effect (subject 1. efference copy $=3.92^{\circ}$, proprioception $=1.68^{\circ}$. subject 2 , efference copy $=2.68^{\circ}$, proprioception $=1.04^{\circ}$ ). The two subjects with statistically significant within-subjects proprioception effects had objective eye deviations of 10 and $5^{\circ}$, respectively.

In all other subjects, measured proprioception deviations were $<1 \%$. Thus the majority of subjects showed no significant influence of change in proprioception on perceived position; those who did, showed substantially less than the amount predicted by an assumption of equal proprioception and efference copy contributions.

We also did a between-subjects analysis using the mean perceptual effect and the mean eye deviation in each subject. The amount of proprioception-induced deviation was plotted against the objectively measured deviation of the occluded eye, so that proprioception and efference copy could be compared (Fig. 2, bot. tom). Correlation between judged position and eye deviation was 0.54 ; the figure makes it apparent that the reason for the low correlation is a floor effect in the judgment measure. The positive $x$-intercept shows that no effect of proprioception is seen for low amounts of eye deviation; the very shallow slope of the regression line $(0.13)$ means that the quantitative estimate of the threshold for proprioception effects is unreliable. This slope is in remarkably close agreement with the slope found by Gauthier et al. $(1990 \mathrm{a}, \mathrm{b})$, which was 0.165 for the left and right eyes averaged, despite the differences in method and in the range of ocular deviations.

Shallow slope indicates that deviated proprioception, when it has any effect at all, is heavily undervalued in perception. Since we expect effects half as large as the objective deviation, the best estimate of the effect size is obtained by doubling the slope. This yields an estimate that after a threshold region of no effect, about $25.6 \%$ of the proprioception signal affects perception. 
Open-loop pointing. Proprioception effects did not reach statistical significance for any of the individual subjects, chiefly because of the greater variability in the pointing measure.

\section{Comparison of efference copy and proprioception}

The analysis in Fig. 1 predicts that the effects of proprioception, if any, should be opposite in direction to those of efference copy. In all but 1 of the 8 subjects, the effects were either opposite (even if statistically unreliable) or the proprioceptive effect was zero.

The proprioceptive effect can also be compared directly to the corresponding efference copy effect in the same subject by plotting one against the other (Fig. 3). If the proprioception effect is functionally significant, subjects with a large eyepress and a large efference copy effect should have a large proprioception effect as well. Again there is a floor effect in the proprioception measure, but also a positive correlation between subjects in the proprioception and efference copy measures.

Armed with quantitative measures of the slopes of the functions in our proprioception and efference copy conditions, we can now calculate the internal physiological effects of the two sources of extraretinal information. All variables are treated as gains. To combine monocular and binocular effects, we consider

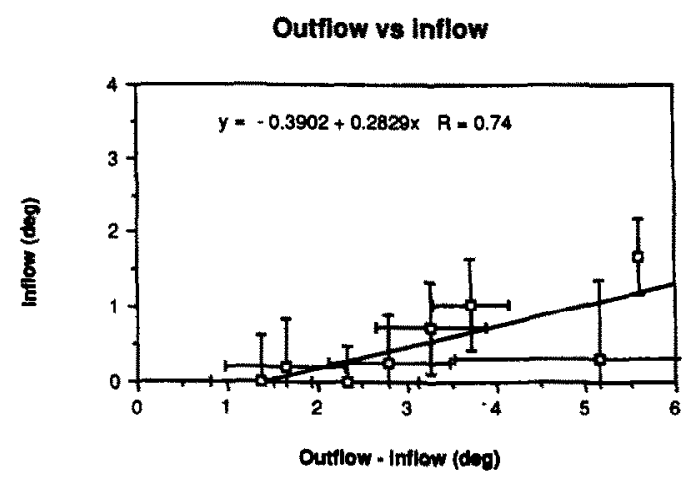

Fig. 3. Efference copy vs proprioception influences on judging. Each subject is represented by one point. Because the experiment's efference copy measure combines effects of efference and proprioception, an estimate of the isolated effect of efference copy is calculated by subtracting data in the proprioception condition from data in the efference copy condition (Outflow-Inflow). This means that the vertical and horizontal axes are not statistically independent; the correlation should be interpreted as a descriptive statistic only. Horizontal error bars are within-subject standard deviations in the efference copy (Outfiow) condition; vertical error bars are within-subject standard deviations in the proprioception (Inflow) condition. An equal contribution of both variables would result in a line through the origin with a slope of 1 . the influences on an imaginary cyclopean eye, equally affected by the two anatomical eyes according to Hering's Law. We use the following nomenclature:

$$
\begin{aligned}
E_{\text {exp }}= & \text { experimentally measured efference } \\
& \text { copy condition } \\
P_{\text {exp }}= & \text { experimentally measured proprio- } \\
& \text { ception condition } \\
E_{\text {int }}= & \text { internal efference copy } \\
P_{\text {int }}= & \text { internal proprioception. }
\end{aligned}
$$

From Fig. 1, the experimental measured efference copy $E_{\text {exp }}$ contain both an efference component and half of the physiological proprioception effect:

$$
E_{\text {exp }}=E_{\text {int }}+P_{\text {int }} / 2 .
$$

The experimentally measured proprioception $P_{\exp }$ is half of the physiological effect, since we deviate only one eye:

$$
P_{\exp }=P_{\text {int }} / 2 \text {. }
$$

Solving equation (1) for $E_{\text {int }}$, and changing signs because the perceptual effects are in the opposite direction from the eyepress, we obtain

$$
E_{\text {int }}=P_{\text {int }} / 2-E_{\text {exp }} \text {. }
$$

Substituting from equation (2) yields

$$
E_{\text {int }}=P_{\text {exp }}-E_{\text {exp }} \text {. }
$$

Solving equation (2) for $\mathrm{P}_{\mathrm{int}}$ yields

$$
P_{\text {int }}=2\left(P_{\text {exp }}\right) \text {. }
$$

Equations (4) and (5) can now be used to find the physiological effects of efference copy and proprioception. Substituting into equation (4),

$$
\mathrm{E}_{\text {int }}=0.13-0.74=-0.61 \text {. }
$$

Thus our estimate of the gain of efference copy in our conditions is about $5 / 8$. The gain is negative because the perceptual effects are in the opposite direction from the eyepress. Substituting into equation (5),

$$
P_{\text {int }}=2(0.13)=0.26 \text {. }
$$

Thus our estimate of the effect of proprioception on perception when no visual context is present is about $1 / 4$.

Efference copy and proprioception effects for both tasks are summarized for all subjects in Fig. 4. Pointing and judging are not statistically different from one another, either for the efference copy condition, comparing the first and second bars in the figure $\left(t_{11}=0.93\right.$, $P=0.74$ ), or for the proprioception condition, 


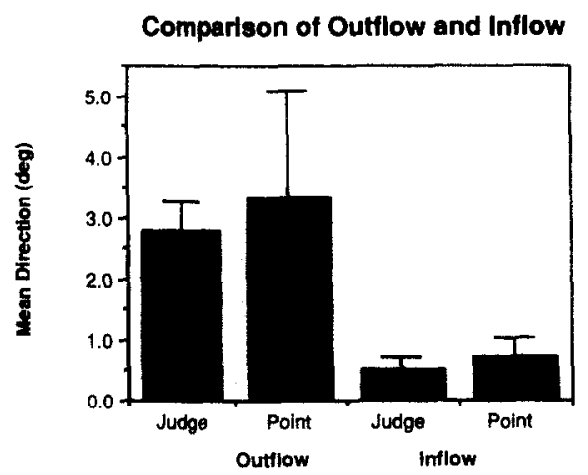

Fig. 4. Summary of effects of efference copy (Outflow) and proprioception (Inflow) on judging and pointing; the error bars represent between-subjects standard error. One outlier subject was omitted from the pointing data shown because of an effect 9 times the average, and 3 times that of the next highest subject. Differences between judging and pointing data are not statistically significant.

comparing the third and fourth bars $\left(t_{12}=0.52\right.$, $P=0.65$ ).

Deviations in judging were significantly larger for efference copy than for proprioception, comparing the first and third bars $t_{14}=4.36$, $P=0.00066$ ). The difference between efference copy and proprioception for the pointing measure, though it is larger than the difference for the judging measure in absolute value, did not reach statistical significance $\left(t_{9}=1.64, P=\right.$ $0.19)$ because of the variability in the efference copy pointing data.

\section{DISCUSSION}

The results obtained in this experiment showed efference copy to be the dominant source of information that informs both perception and visually guided behavior. Proprioception did have an effect, at lease in perception, but the slope of the proprioception function was very shallow-a large change in eye position resulted in only a small change in the proprioception signal. Further, there was a threshold value below which inflow had no effect.

Our proprioception results can best be compared to those obtained by Gauthier et al. $(1990 \mathrm{a}, \mathrm{b})$. The subject who was run both in Gauthier's laboratory and in ours showed a measurable effect of proprioception and of course a larger effect of efference copy as well in Gauthier's apparatus (Gauthier \& Stark, unpublished result, 1989). In our experiment, with smaller eye deviations, this subject's proprioception effect was also small (mean $=0.2^{\circ}$, $t_{29}=0.41 \mathrm{NS}$ ). The overall agreement of our results and those of Gauthier et al, across subjects shows that Gauthier's effect is robust and repeatable.

\section{Remainder effect}

The sum of our calculated physiological efference copy and proprioception effects is $0.61+$ $0.26=0.87$, implying that in an unstructured field the combined effects of proprioception and efference copy still do not completely register the eye deviation. This may explain the tendency of subjects to underestimate the amount of rotation of their own eyes away from the straight-ahead position. This tendency was studied quantitatively by Morgan (1978), who had subjects fixate an eccentric target and then estimate whether another target was left or right of center (azimuth) in an otherwise unstructured field. There was a linear relationship between eccentricity of the fixation target and error in judging the straight-ahead direction. Combining Morgan's data for left and right deviations, we performed a linear regression and found that the linear component accounted for $97 \%$ of the variance in her function. The maximum deviation of straight-ahead in Morgan's data was $5.3^{\circ}$ for a fixation deviation of $42^{\circ}$; straight ahead estimates deviated in the same direction as the eye. Morgan did not calculate the slope of this function, but from the published data we calculate a slope of 0.13 for the function of fixation eccentricity vs straight-ahead deviation. This slope is an estimate of the missing gain in the oculomotor registration of ocular eccentricity; adding this value to our sum of proprioception and efference copy yields a total of 1.00 . Thus our data account precisely for the magnitude of the straight-ahead illusion found by Morgan.

Hill (1972) estimated the deviation between oculomotor registration of visual direction and acutal direction with another method, constrasting deviations estimated during eye turn and during head turn with the eyes in primary position. His estimate of the under-registration of ocular deviation was similar to that of Morgan, so that our data account quantitatively for this result also.

Comparison with other estimates of extraretinal signals

Our estimate of the magnitude of the extraretinal signal, compounded of both 
proprioception and efference copy, can be compared with other estimates made in uniform fields with other methods. The gain of extraretinal signals during saccades can be calculated from data of Grüsser, Krizic and Weiss (1987). Subjects fixated a bright light to create an afterimage in darkness, and then performed repeated saccades to alternate fixation between two auditory targets. They then estimated the spatial separation of the resting points of the afterimage when the eye was in the left versus the right position. At their slowest saccade rate of about one saccade every $667 \mathrm{msec}$, average separation of pointing estimations in the two positions was about $22^{\circ}$ across saccades of $46^{\circ}$. These numbers cannot be used directly to calculate gain, however, because slope of their line comparing pointer positions with target separations using real light stimuli was only between 0.8 and 0.95 . Using the mean of this range as a correction factor, we calculate the gain of the extraretinal signal to be 0.55 . Extrapolation from the data of Grüsser et al. indicates that gain might be slightly higher with longer intersaccadic intervals.

For pursuit eye movements the estimates are more complicated because there must be a stimulus for pursuit as well as an open-loop target. Pola and Wyatt (1989) isolated extraretinal contributions to movement perception by presenting an outline square stabilized on the retina. Any apparent movement of the square must be due to extraretinal influences combining proprioception and efference copy, because real motions on the retina are cancelled mechanically. To elicit smooth tracking eye movements, retinal slip was produced by stepping a bar back and forth within the square. The observer, attempting to fixate the bar, generated smooth pursuit movements because the bar did not move far enough from the center of fixation to elicit saccades. Apparent motions under the open-loop condition were compared with subsequent perceived motions under normal closed-loop conditions by a method of adjustment.

As was the case for saccades, the open-loop gain was dependent on the frequency of oscillation of the bar (and of the pursuit eye movements). At the slowest oscillation frequency of $0.5 \mathrm{~Hz}$, the average gain for three subjects was 0.79 , as compared to our composite gain estimate during fixation of 0.87 . The reason for our higher estimate may be that the gain of the extraretinal signal tends to decrease with more frequent eye movement. Pola and Wyatt's gain decreased to less than 0.4 at a frequency of $2 \mathrm{~Hz}$. They model the transfer function as a moderately damped second-order system.

\section{Other types of proprioception}

These conclusions hold for proprioception sources (i.e. spindle receptors) that signal muscle length. There remains one further class of proprioception that might account for some of our results. Muscle receptors such as Golgi tendon organs that respond to tension of the extraocular muscles, rather than their length, might be stimulated by an eyepress with the viewing eye even though the position of the eye in the orbit does not change. The increase in muscle tension required to counteract the rotational force of the eyepress might stimulate such receptors, giving a proprioception signal (mis)informing the CNS about eye rotation.

If tension receptors contributed actively to either perception or visually guided behavior, however, several dramatic psychophysical effects would ensue. Large mislocalization of targets flashed during saccadic eye movements should occur, because the agonist extraocular muscles develop maximal tension during all moderate and large sized saccades. During a saccade, tension receptors would generate a signal informing the CNS that targets should be localized at or beyond the extreme of the gaze field in the direction of a saccade. This effect would be seen only during approximately the first $2 / 3$ of the saccade duration, and would not infuence localization at any other time.

It has been known for some time, however, that such mislocalizations do not occur, in agreement with Cook and Stark's (1968) conclusion that during a saccade the control is open-loop to both vision and proprioception. Rather, localization changes relatively little during a saccadic eye movement, and most of the correction of spatial values takes place before or after the movement (Bischof \& Kramer, 1968; Matin, 1972, 1986). Though there is some disagreement about the dynamics of the correction function, large jumps in apparent position occurring only during the initial phases of a saccade have never been observed. Thus extraocular muscle tension receptors can be excluded as significant contributors to psychophysical localization. 


\section{CONCLUSIONS}

The function of proprioception in perception and visual-motor coordination, then, seems to be as a backup for the principal influence of efference copy signals, though it provides a significant supplement to the registration of eye position. Efference copy and proprioception together still yield an under-registration of the eccentricity of eye position. Proprioception also seems to be important in development.

This paper has addressed the contributions of proprioception and efference copy only in an unstructured visual field. Quantitative evaluation of the two influences in the structured visual fields of everyday perception remains to be done.

Acknowledgements - We thank Gabriel Gauthier for getting us interested in proprioception and for discussion of the experiments, and Lisa Kerr for able assistance in running subjects and analyzing data. Partially supported by a faculty research grant from the University of California to B.B., and from a cooperative agreement from NASA/Ames Research Center with L.W.S.

\section{REFERENCES}

Ashton, J. A., Boddy, A. \& Donaldson, I. M. L. (1984). Directional selectivity in the responses of units in cat primary visual cortex to passive eye movement. Neuroscience, 13, 653-662.

Bischof, N. \& Kramer, B. (1968). Untersuchengen und Úberlegungen zur Richtungswahrnehmung bei willkürlichen sakkadischen Augenbewegungen. Psychologische Forschung, 32, 195-218.

Bridgeman, B. (1979), Adaptation and the two-visualsystems hypothesis. Behavioral and Brain Science, 2, 84-85.

Bridgeman, B. (1986). Multiple sources of outflow in processing spatial information. Acta Psychologica, 63, 35-48.

Bridgeman, B. \& Delgado, D. (1984). Sensory effects of eyepress are due to efference. Perception and Psychophysics, 36, 482-484.

Bridgeman, B. \& Fishman, R. (1985). Dissociation of corollary discharge from gaze direction does not induce a straight-ahead shift. Perception and Psychophysics, 37, 523-528.

Bridgeman, B. \& Graziano, J. A. (1989). Effect of context and efference copy on visual staight ahead. Vision Research, 29, 1729-1736.

Bridgeman, B. \& Stark, L. (1981). Efferent copy and visual direction. Investigative Ophthalmology and Visual Science, Suppl., 20, 55.

Bridgeman, B., Kirch, M. \& Sperling, A. (1981). Segregation of cognitive and motor aspects of visual function using induced motion. Perception and Psychophysics, 29, 336-342.

Brindley, G. S., Goodwin, G. M., Kulikowski, J. J. \& Leighton, D. (1976). Stability of vision with a paralysed eye. Journal of Physiology, 258, 65-66.
Buissert, P. \& Maffei, L. (1977). Extraocular proprioceptive projections to the visual cortex. Experimental Brain Research, 28, 421-425.

Cook, G. \& Stark, L. (1968). The human eye-movement mechanism: Experiments, modeling, and model testing. Archives Ophthalmology, 79, 428-436.

Descartes, R. (1972). Treatise on man. Hall, T. S. (Ed. and Trans.), Cambridge, Mass.: Harvard University Press (Originally published 1665 ).

Donaldson, I. \& Long, A. C. (1980). Interaction between extraocular proprioceptive and visual signals in the superior colliculus at the cat. Journal of Physiology, 298. 85-110.

Fiorentini, A. \& Maffei, L. (1977). Instability of the eye in the dark and proprioception. Nature, 269, 330-331.

Gauthier, G., Nommay, D. \& Vercher, J.-L. (1990). Ocular muscle proprioception and visual localization of targets in man. Brain, 113, 1857-1871.

Gauthier, G., Nommay, D. \& Vercher, J.-L. (1990). The role of ocular muscle proprioception in visual localization of targets. Science, 249, 58-61.

Graves, A., Trotter, Y. \& Fregnac, Y. (1987). Role of extraocular muscle proprioception in the development of depth perception in cats. Journal of Neurophysology, 85 , 816-831.

Grüsser, O.-J., Krizic, A. \& Weiss, L.-R. (1987). Afterimage movement during saccades in the dark. Vision Research, 27, 215-226.

Hein, A. \& Diamond, R. (1983). Contribution of eye movement to the representation of space. In Hein, A. \& Jeannerod, M. (Eds), Spatially oriented behavior. New York: Springer.

von Helmholtz, H. (1866). Handbuch der Physiologischen Optik Bd. III. Leipzig: Voss. English translation 3rd edn. 1925. Southall, J. P. C. (Ed.), A treatise on physiological optics (Vol. 3). New York: Dover, 1963.

Hering, E. (1968). Die Lehre vom Binokularen Sehen. Leipzig: Engelman. Translated by Bridgeman, B. as The theory of binocular vision, Bridgeman, B. \& Stark, L. (Eds). New York: Plenum Press, 1977.

Hill, A. L. (1972). Direction constancy. Perception and Psychophysics, 11, 175-178.

Ilg, U. J., Bridgeman, B. \& Hoffman, K. P. (1989). Influence of mechanical disturbance on oculomotor behavior Vision Research, 29, 545-551.

Kashii, S., Matsui, Y., Honda, Y., Ito, J., Sasa, M. \& Takaori, S. (1989). The role of extraocular proprioception in vestibulo-ocular reflex of rabbits. Investigative Ophthalmology and Visual Science, 30, 2258-2264.

Kornmüller, A. E. (1931). Eine experimentelle Anästhesie der äusseren Augenmuskeln am Menschen und ihre Auswirkungen. Journal für Psychologie und Neurologie (Leipzig), 41, 354-366.

Ludvigh, E. (1952). Possible role of proprioception in the extraocular muscles. Archives of Ophthalmology, 48, 436-441.

Matin, L. (1972). Eye movements and perceived visual direction. In Jameson, D. \& Hurvich, L. (Eds), Handbook of sensory physiology (Vol. 7/4). Heidelberg: Springer.

Matin, L. (1986). Visual localization and eye movements. In Boff, K. R., Kaufman, L. \& Thomas, J. P. (Eds), Handbook of perception and human performance, Vol. I. Sensory processes and perception. New York: Wiley.

Matin, L., Picoult, E., Stevens, J. K., Edwards, Jr M. W., Young, D. \& MacArthur, R. (1980). Visual context dependent mislocalizations under curare-induced 
partial paralysis of the extraocular muscles. Investigative Ophthalmology and Visual Science, Suppl., 19, 81.

Matin, L., Picoult, E., Stevens, J. K., Edwards, Jr M. W., Young, D. \& MacArthur, R. (1982). Oculoparalytic illusion: Visual-field dependent spatial mislocalizations by humans partially paralyzed with curare. Science, 216, 198-201.

Milleret, C. (1987). Projections centrales des afférences proprioceptives issues des muscles extraocularies chez les vertébrés. Quelques rôles fonctionnels possibles dans le contrôle de l'oculomotricité, la perception visuelle et l'orientation corporelle. Agressologie, 28, 917-924.

Mittelstaedt, H. (1990). Basic solutions to the problem of head-centric visual localization. In Warren, R. \& Wertheim, A. (Eds), Perception and control of self-motion. Hillsdale, N.J.: Erlbaum.

Morgan, C. L. (1978). Constancy of egocentric visual direction. Perception and Psychophysics, 23, 61-68.

Paillard, J. (1987). Cognitive versus sensorimotor encoding of spatial information. In Ellen, P. \& Thinus-Blanc, C. (Eds), Cognitive processes and spatial orientation in animal and man. Dordrecht: Nijhoff.

Pola, J. \& Wyatt, H. J. (1989). The perception of target motion during smooth pursuit eye movements in the open-loop condition: Characteristics of retinal and extraretinal signals. Vision Research, 29, 471-483.

Robinson, D. A. (1964). The mechanies of human saccadic eye movement. Journal of Physiology, 174, 245-264.

Sherrington, C. S. (1898). Further note on the sensory nerves of the eye muscles. Proceedings of the Royal Society, 64, 120-121.

Sherrington, C. S. (1918). Observations on the sensual role of the proprioceptive nerve supply of the extrinsic ocular muscles. Brain, 41, 332-343.

Siebeck, R. (1954). Wahrnehmungsstörung und Stórungswahrenhmung bei Augenmuskelláhmungen. von Graefes Archiv fur Opthalmologie, 155, 26-34.
Skavenski, A. A. (1972). Inflow as a source of extraretinal eye position information. Vision Research, 12, 221-229.

Skavenski, A. A., Haddad, G. \& Steinman, R. M. (1972). The extraretinal signal for the visual perception of direction. Perception and Psychophysics, 11, 287-290.

Sperry, R. W. (1950). Neural basis of the spontaneous optokinetic response produced by visual inversion. Journal of Comparative Psychology and Physiology, 43, 482-489.

Stark, L. \& Bridgeman, B. (1983). Role of corollary discharge in space constancy. Perception and Psychophysics, $34,371-380$.

Stark, L., Vossius, G. \& Young, L. R. (1962). Predictive control of eye tracking movements. Institute of Radio Engineers Transactions on Human Factors in Electronics, HFE-3, 52-57.

Steinbach, M. J. (1987). Proprioceptive knowledge of eye position. Vision Research, 10, 1737-1744.

Steinbach, M. J. \& Smith, D. R. (1981). Spatial localization after strabismus surgery: Evidence for proprioception. Science, 213, 1407-1409.

Stevens, J. K., Emerson, R. C., Gerstein, G. L., Kallos, T., Neufeld, G. R., Nichols, C. W. \& Rosenquist, A. C. (1976). Paralysis of the awake human: Visual perceptions. Vision Research, 16, 93-98.

Trotter, Y., Fregnac, Y. \& Buisseret, P. (1987). The period of susceptibility of visual cortical binocularity to unilateral proprioceptive deafferentation of extraocular muscles. Journal of Neurophysiology, 58, 795-815.

von Holst, E. \& Mittelstaedt, H. (1950). Das Reafferenzprinzip. Wechselwirkungen zwischen Zentralnervensystem und Peripherie. Naturwissenschaften, 37, 464-476.

Wong, E. \& Mack, A. (1981). Saccadic programming and perceived location. Acta Psychologica, 48, 123-131. 